\title{
A Multi Dimensional Stochastic Differential Equation Model
}

\author{
N. Hema ${ }^{1} \&$ Dr. A. Jayalakshmi ${ }^{2}$ \\ ${ }^{I}$ Research Scholar, SCSVMV, Kanchipuram, India. \\ ${ }^{2}$ Prof. \& Head, Dept of Mathematics, SCSVMV, Kanchipuram,. India.
}

\begin{abstract}
In this paper, we discuss a mulit dimensional stochastic differential equation model where the underlying stochastic process is a jump-diffusion process. The stochastic differential equation is represented as a Partial Integro Differential Equation(PIDE) using the Fokker Planck equation. The solution of the PIDE is obtained by the method of finite differences. The consistency, the convergence of the solution and the stability of the finite difference scheme are discussed.
\end{abstract}

Keywords: jump-diffusion process, partial integro differential equation, stability of the finite difference scheme, consistency and convergence of a numerical solution.

\section{Introduction}

Some physical problems may involve multiple state variables and multiple sources of random disturbances. In such cases the multi dimensional stochastic differential equation is useful.We recall the results for a multi dimensional stochastic process as stated in Hanson[5].

Let $\bar{X}(\mathrm{t})=\left[\mathrm{X}_{\mathrm{i}}(\mathrm{t})\right]$ be a vector stochastic process of dimension $\mathrm{n}_{\mathrm{x}}$ and $\bar{W}(\mathrm{t})=\left[\mathrm{W}_{\mathrm{i}}(\mathrm{t})\right]$ be a Wiener process of dimension $\mathrm{n}_{\mathrm{w}}$. If $\mathrm{f}$ and $\mathrm{g}$ are functions depending on the vector stochastic process $\bar{X}(t)$ then a multi dimensional SDE is of the form

$$
\mathrm{d} \bar{X}(\mathrm{t})=\mathrm{f}(\bar{X}(\mathrm{t}), \mathrm{t}) \mathrm{dt}+\mathrm{g}(\bar{X}(\mathrm{t}), \mathrm{t}) \mathrm{d} \bar{W}(\mathrm{t})
$$

The multi dimensional SDE with jump is of the form

$$
\mathrm{d} \bar{X}(\mathrm{t})=\mathrm{f}(\bar{X}(\mathrm{t}), \mathrm{t}) \mathrm{dt}+\mathrm{g}(\bar{X}(\mathrm{t}), \mathrm{t}) \mathrm{d} \bar{W}(\mathrm{t})+\mathrm{h}(\bar{X}(t), \mathrm{t} \bar{Q}) \mathrm{d} \bar{P}(\bar{X}(t), t, \bar{Q})
$$

where $\bar{P}(\bar{X}(t), t, \bar{Q})$ is a $\mathrm{n}_{\mathrm{p}}$ dimensional vector state dependent jump process and $\bar{Q}=\left[\mathrm{Q}_{\mathrm{i}}\right]$ is the vector notation for the jump amplitude mark. The coefficient $\mathrm{h}$ of the jump amplitude process has the form $\mathrm{h}(\bar{X}(t), \mathrm{t}$ $\left.\bar{Q})=\left[\mathrm{h}_{\mathrm{ij}} \bar{X}(t), \mathrm{t}, \mathrm{Q}_{\mathrm{j}}\right)\right]$ such that the $\mathrm{j}^{\text {th }}$ component depends only on the $\mathrm{j}^{\text {th }}$ jump mark $\mathrm{Q}_{\mathrm{j}}$.

Ito's chain rule in multi dimensions

If $\bar{Y}(\mathrm{t})$ is twice continuously differentiable in the space variables and once in $\mathrm{t}$ then the chain rule is:

$$
\mathrm{d} \bar{Y}(\mathrm{t})=\bar{F}(\mathrm{t})+\bar{f}^{\mathrm{T}} \nabla_{x}[\bar{F}]+\frac{1}{2} \mathrm{~g} R^{\prime} \mathrm{g}^{\mathrm{T}}: \nabla_{x}\left[\nabla_{x}^{\mathrm{T}}[\bar{F}]\right](\bar{X}(\mathrm{t}), \mathrm{t}) \mathrm{dt}+\int_{Q} \nabla^{T}[\bar{F}] \mathrm{P} .
$$

Here $\nabla_{x}[\bar{F}]=\left[\frac{\partial F_{i}}{\partial x_{i}}\right]$ is the state - space gradient and $\nabla_{x}{ }^{\mathrm{T}}[\bar{F}]$ is its transpose.

Also $\nabla_{x}\left[\nabla_{x}{ }^{\mathrm{T}}[\bar{F}]\right]=\left[\frac{\partial^{2} F_{i}}{\partial x_{i} \partial x_{j}}\right]$ and $\mathrm{R}^{\prime}$ is a correlation matrix $\mathrm{R}^{\prime}=\left[\rho_{\mathrm{ij}}\right]_{\mathrm{nw}}$ xnw and A:B is the double product $\mathrm{A}: \mathrm{B}=$ $\sum_{i=1}^{n} \sum_{j=1}^{n} A_{i j} B_{i j}=\operatorname{tr}\left(\mathrm{AB}^{\mathrm{T}}\right)$.

The use of Fokker Planck or backward Kolmogorov equation in multi dimensions is the PIDE that may be derived from equation (1) and Ito's chain rule. It is of the form:

Let $\bar{X}\left(\mathrm{t}_{\mathrm{o}}\right)=\bar{x}_{\mathrm{o}}$ and $\mathrm{u}\left(\bar{x}_{\mathrm{o}}, \mathrm{t}_{\mathrm{o}}\right)=\mathrm{E}\left(\mathrm{v}\left(\bar{X}(\mathrm{t}) \mid \bar{X}\left(\mathrm{t}_{\mathrm{o}}\right)=\bar{x}_{\mathrm{o}}\right)\right.$. Then u satisfies the partial differential equation:

$$
\begin{gathered}
0=\frac{\partial u}{\partial t}\left(\bar{x}_{\mathrm{o}}, \mathrm{t}_{\mathrm{o}}\right)+\mathrm{f}\left(\bar{x}_{\mathrm{o}}, \mathrm{t}^{\mathrm{T}}{ }_{\mathrm{o}}\right)\left[\nabla_{x}\left(\bar{x}_{\mathrm{o}}, \mathrm{t}_{\mathrm{o}}\right)\right]+\frac{1}{2} \mathrm{gR}^{\prime} \mathrm{g}^{\mathrm{T}}:\left[\nabla_{x}\left[\nabla^{\mathrm{T}}{ }_{\mathrm{x}}\left(\bar{x} \mathrm{o}, \mathrm{to}_{0}\right)\right]\right]+ \\
\sum_{j=1}^{n} \hat{\lambda}_{j}\left(\bar{x}_{\mathrm{o}}, \mathrm{t}_{\mathrm{o}}\right) \int_{Q}\left[u\left(x+h\left(\overline{x_{o}}, t, q_{j}\right)\right)-u\left(\bar{x}_{o}, t\right)\right] \varphi\left(q_{j}\right) d q_{j} .
\end{gathered}
$$

\section{The proposed multi dimensional SDE model :}

Suppose a stochastic process $X$ depends on several mutually independent variables $X_{1}, X_{2}, \ldots \ldots . X_{k}$. Then $\mathrm{X}$ is a vector process having $\mathrm{k}$ components. This process is written as $\bar{X}$. We assume that $\bar{X}$ satisfies a SDE with jumps. Let $\bar{Q}$ be the jump vector. Each jump component is represented in the form $\mathrm{Q}_{\mathrm{j}}=\mathrm{Q}_{\mathrm{j}}{ }^{+}-Q i^{-}$

Define $\mathrm{Q}_{\mathrm{i}}^{+}=\max \left(\mathrm{Q}_{\mathrm{i}}, 0\right)$ and $Q i^{-}=\max \left(-\mathrm{Q}_{\mathrm{i}}, 0\right)$. Then $Q i^{+}$and $Q i^{-}$are respectively the positive and negative parts of $\mathrm{Q}_{\mathrm{i}}$. We shall assume suitable statistical distributions for $Q i^{+}$and $Q i^{-}$for each i. Let $\gamma_{\mathrm{i}}, \lambda_{\mathrm{i}}$ be the parameters of the distribution of $\mathrm{Q}_{\mathrm{i}}^{+}$and $\beta_{\mathrm{i}}, \mu_{\mathrm{i}}$ be the parameters of the distribution of $Q i^{-}$. Let $\Phi^{+}$and $\Phi^{-}$be the joint densities of $\bar{Q}^{+}$and $\bar{Q}^{-}$respectively. We represent the process as $\bar{X}=\bar{Z}+\bar{Q}^{+}-\bar{Q}^{-}$where the component $\mathrm{Zi}$ of $\bar{Z}$ is normal in $\left[\beta_{\mathrm{i}}, \gamma_{\mathrm{i}}\right]$.

Now we construct a stochastic differential equation in the form 


$$
\mathrm{d} \bar{X}(\mathrm{t})=\mathrm{f}(\bar{X}(\mathrm{t}), \mathrm{t}) \mathrm{dt}+\mathrm{g}(\bar{X}(\mathrm{t}), \mathrm{t}) \mathrm{d} \bar{W}(\mathrm{t})+\mathrm{h}_{1}\left(\bar{X}(t), \mathrm{t}, \bar{Q}^{+}\right) \mathrm{d} \bar{P}^{+}+\mathrm{h}_{2}\left(\bar{X}(t), \mathrm{t}, \bar{Q}^{-}\right) \mathrm{d} \bar{P}^{-}
$$

where the components $\mathrm{Q}_{\mathrm{i}}^{+}$and $Q i^{-}$of $\bar{Q}$ have suitable distributions in the intervals $\left[\gamma_{\mathrm{i}}, \delta_{\mathrm{i}}\right]$ and $\left[\alpha_{\mathrm{i}}, \beta_{\mathrm{i}}\right]$ respectively. Using the FP equation the $\mathrm{SDE}$ (3) can be written in its equivalent form as a PIDE as

$$
\begin{aligned}
& 0=\frac{\partial u}{\partial t}\left(\bar{x}_{0}, \mathrm{t}_{\mathrm{o}}\right)+\mathrm{f}^{\mathrm{T}}\left(\bar{x}_{\mathrm{o}}, \mathrm{t}^{\mathrm{T}}{ }_{\mathrm{o}}\right)\left[\nabla_{x}\left(\bar{x}_{\mathrm{o}}, \mathrm{t}_{\mathrm{o}}\right)\right]+\frac{1}{2} \mathrm{gR}^{\prime} \mathrm{g}^{\mathrm{T}}:\left[\nabla_{x}\left[\nabla^{\mathrm{T}}{ }_{\mathrm{x}}(\bar{x} \mathrm{o}, \mathrm{to})\right]\right]+ \\
& \hat{\lambda} \int_{\tilde{Q}_{+}+}\left[\bar{u}\left(x+h\left(\overline{x_{0}}, t, \bar{q}\right)\right)-\bar{u}\left(\overline{x_{0}}, t\right)\right] \Phi^{+}(\bar{q}+) d \overline{q^{+}}+ \\
& \hat{\mu} \int_{\widetilde{Q}^{-}}\left[\left[\bar{u}\left(x+h\left(\overline{x_{o}}, t, \bar{q}\right)\right)-\bar{u}\left(\overline{x_{o}}, t\right)\right]\right] \Phi^{-}\left(\overline{q^{-}}\right) d \overline{q^{-}}
\end{aligned}
$$

The symbol $\hat{Q}$ in the integral terms denote k-dimensional integrals and the symbol $d \bar{q}$ denotes $\mathrm{dq}_{1} \mathrm{dq}_{2} \ldots . . . \mathrm{dq}_{\mathrm{k}}$. The partial derivative terms having coefficients $\mathrm{f}$ and $\mathrm{g}$ have the following meaning: Let $\mathrm{f}=\left(\mathrm{f}_{1,} \mathrm{f}_{2,}, \ldots . \mathrm{f}_{\mathrm{k}}\right)$. Then $\mathrm{f}^{\mathrm{T}}\left(\bar{x}_{\mathrm{o}}, \mathrm{t}^{\mathrm{T}}{ }_{\mathrm{o}}\right)\left[\nabla_{x} u\right]=\mathrm{f}_{\mathrm{i}} \frac{\partial u}{\partial x_{1}}+\mathrm{f}_{\mathrm{i} 2} \frac{\partial u}{\partial x_{2}}+\ldots . .+\mathrm{f}_{\mathrm{k}} \frac{\partial u}{\partial x_{k}}$.

Taking $\mathrm{g}=\left(\mathrm{g}_{\mathrm{i} 1}, \mathrm{~g}_{\mathrm{i} 2}, \ldots . . \mathrm{g}_{\mathrm{ik}}\right)$ and $\mathrm{R}^{\prime}=\left[\rho_{\mathrm{ij}}\right]$ a correlation matrix of order $\mathrm{k} \mathrm{x} \mathrm{k}$, the product $\mathrm{gR}^{\prime} \mathrm{g}^{\mathrm{T}}$ is the scalar $\sum_{i=1}^{k} g_{i} \sum_{j=1}^{k} g_{j} \rho_{i j}$. Thus $\mathrm{gR}^{\prime} \mathrm{g}^{\mathrm{T}}:\left[\nabla_{x}\left[\nabla^{\mathrm{T}} \mathrm{x} \mathrm{u}\right]\right.$ is the expression $\sum_{l=1}^{k} g_{l} \sum_{j=1}^{k} g_{j} \rho_{l j}\left(\frac{\partial^{2} u}{\partial x_{1}^{2}}+\frac{\partial^{2} u}{\partial x_{2}^{2}}+\ldots .+\frac{\partial^{2} u}{\partial x_{k}^{2}}\right)$.

Let $\mathrm{H}_{1}=\sum_{j=1}^{k} g_{j} \rho_{l j}$.

Let $\mathrm{D}=\mathrm{f}_{1} \frac{\partial u}{\partial x_{1}}+\mathrm{f}_{2} \frac{\partial u}{\partial x_{2}}+\ldots . .+\mathrm{f}_{\mathrm{k}} \frac{\partial u}{\partial x_{k}}+\frac{H_{1}}{2} \frac{\partial^{2} u}{\partial x_{1}^{2}}+\frac{H_{2}}{2} \frac{\partial^{2} u}{\partial x_{2}^{2}}+\ldots .+\frac{H_{k}}{2} \frac{\partial^{2} u}{\partial x_{k}^{2}}$ and $\mathrm{I}=\mathrm{I}_{1}-\mathrm{I}_{2}$.

\section{The finite difference scheme}

We use the implicit - explicit scheme as adopted by R.Cont for a two dimensional process[1]. We use the scheme for the $\mathrm{n}$ dimensional process. The derivative terms are replaced by finite difference approximations at time step $n+1$ and the integral terms by approximate summations at time step $n$, for any $n=0,1,2 \ldots \ldots \mathrm{N}-1$.

Let $\mathrm{C}_{0}^{\infty}$ be the space of continuously differentiable functions with a norm $\|$.

Let $\mathrm{u} \in \mathrm{C}_{0}^{\infty}\left([0, \mathrm{~T}] \times \mathrm{R}^{\mathrm{k}}\right)$ be continuously differentiable as many times as required for $\mathrm{t} \in[0, \mathrm{~T}]$ and $\bar{x} \in \mathrm{R}^{\mathrm{k}}$. The first and second order partial derivatives of $\mathrm{u}$ with respect to each component $\mathrm{x}_{\mathrm{i}}$ of $\bar{x}$ are discretized as follows[4]:

$$
\begin{array}{r}
\text { For }\left(\mathrm{t}_{\mathrm{n}}, \mathrm{x}_{\mathrm{i}}\right) \in[0, \mathrm{~T}] \mathrm{xR}, \quad\left(\frac{\partial u}{\partial x}\right)_{i}=\frac{\left(u_{i+1}-u_{i}\right)}{\Delta x} ; \\
\left(\frac{\partial^{2} u}{\partial x^{2}}\right)_{\mathrm{i}}=\frac{\left(u_{i+1}-2 u_{i}+u_{i-1}\right)}{(\Delta x)^{2}} .
\end{array}
$$

Moreover an integral of the form $\int_{\gamma}^{\infty}[u(x+h(x, t, q), t)-u(x, t)] \varphi(q) \mathrm{dq}$ is approximated to a sum $\sum_{j=K_{1}}^{k_{2}} \varphi_{1}(q)\left(u_{k+j}-u_{j}\right)$ using trapezoidal quadrature formula after replacing the limits of integration by a suitable bounded interval, say, $[\gamma, \delta]$. The following notations are used to write the partial derivative approximations when $\mathrm{u} \in \mathrm{C}_{0}^{\infty}\left([0, \mathrm{~T}] \mathrm{x} \mathrm{R}^{\mathrm{k}}\right)$.

The cartesian product of the intervals of the form $\left[\alpha_{i}, \delta_{i}\right]$ is denoted as $[\alpha, \delta]$ and the region $[0, T] x$ $[\alpha, \delta]$ is divided into sub regions by introducing meshes of width $\Delta \mathrm{t}$ for $[0, \mathrm{~T}]$ and $\Delta \mathrm{x}_{\mathrm{i}}$ for each interval $\left[\alpha_{\mathrm{i}}, \delta_{\mathrm{i}}\right]$. Further,

$$
\begin{aligned}
& \text { for, } \overline{x_{l l}^{n}}=\left(\mathrm{t}_{\mathrm{n}}, \mathrm{x}_{\mathrm{i} 1}, \mathrm{x}_{\mathrm{i} 2} \ldots . . . \mathrm{x}_{\mathrm{ik} .}\right) \in[0, \mathrm{~T}] \mathrm{x}[\alpha, \delta] \\
& \left(\frac{\partial u}{\partial x_{i_{l}}}\right)_{\overline{x_{l}}}=\frac{\left(u_{i_{1}, \ldots, i_{l}+1, \ldots i_{k}}^{n}-u_{i_{1}, \ldots i_{l}, i_{k}}^{n}\right)}{\Delta x_{i_{l}}} \text { where } i_{l}=\mathrm{i} 1, \mathrm{i} 2, \ldots \ldots \ldots \text { ik. }
\end{aligned}
$$

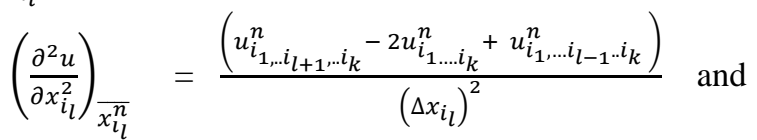

$$
\begin{aligned}
& \left(\frac{\partial u}{\partial t}\right)_{\overline{x_{l}}}=\frac{\left(u_{i_{1}, \ldots i i_{k}}^{n+1}-u_{i_{1}, i_{k}}^{n}\right)}{\Delta t} .
\end{aligned}
$$

Let $\left\{u_{i_{1} i_{2} \ldots i_{k}}^{n}\right\}$ be the solution of the numerical scheme to be defined. Let $K 1_{i_{l}}^{+}$and $K 2_{i_{l}}^{+}$be real numbers such that $\left[\gamma_{\mathrm{i}}, \delta_{\mathrm{i}}\right]$ is contained in $\left.\left[K 1_{i_{l}}^{+}-1 / 2\right) \Delta \mathrm{x},\left(\mathrm{K}_{2}^{+}+1 / 2\right) \Delta \mathrm{x}\right]$ and $K 1_{i_{l}}^{-}, K 2_{i_{l}}^{-}$be such that $\left[\alpha_{\mathrm{i}}, \beta_{\mathrm{i}}\right]$ is contained in $\left[\left(\mathrm{K}_{1}^{-}-1 / 2\right) \Delta \mathrm{x},\left(\mathrm{K}_{2}^{-}+1 / 2\right) \Delta \mathrm{x}\right]$.

Now the partial integro differential equation (4) gives rise to a system of linear equations

$\frac{\left(u_{i_{1} i_{2} \ldots i_{k}}^{n+1}-u_{i_{1} i_{2} \ldots i_{k}}^{n}\right)}{\Delta t}+\sum_{l=1}^{k} f_{l} \frac{\left(u_{i_{1 . . i_{l+1} \cdot i_{k}}}^{n}-u_{i_{1} \ldots i_{l . i_{k}}}^{n}\right)}{\Delta x_{i_{l}}}+\sum_{l=1}^{k} \frac{H_{l}}{2} \frac{\left(u_{i_{1 . . i}}^{n} i_{l+1 . i_{k}}-2 u_{i_{1}, i_{l} \cdot i_{k}}^{n}+u_{i_{1} \cdot i_{l} \cdot i_{l}}^{n}\right)}{\left(\Delta x_{i_{l}}\right)^{2}}=\mathrm{J}_{2}-\mathrm{J}_{1}, \ldots \ldots$

$\mathrm{n}=0,1, \ldots \mathrm{N}-1 ; i_{l}=1,2 \ldots \ldots \mathrm{k} ; u_{i_{l}}{ }^{\mathrm{n}+1}=0$ for $i_{l}>\mathrm{M}$ and with initial condition

$\mathrm{u}_{0}^{0}=\mathrm{x}_{0}$ 
Here $\mathrm{I}_{1}=\hat{\lambda} \int_{\ddot{Q^{+}}}\left[\bar{u}\left(x+h\left(\overline{x_{0}}, t, \bar{q}\right)\right)-\bar{u}\left(\overline{x_{0}}, t\right)\right] \Phi^{+}(\bar{q}+) d \overline{q^{+}}$and

$\mathrm{I}_{2}=\hat{\mu} \int_{\hat{Q}-}\left[\left[\bar{u}\left(x+h\left(\overline{x_{o}}, t, \bar{q}\right)\right)-\bar{u}\left(\overline{x_{o}}, t\right)\right]\right] \Phi^{-}\left(\overline{q^{-}}\right) d \overline{q^{-}}$, where the symbols

$\hat{Q}+, \hat{Q}-$ denote the regions of integration as $[\gamma, \delta]$ and $[\alpha, \beta]$. Also $\hat{\lambda}$ and $\hat{\mu}$

are parameters of the distributions of $\bar{Q}^{+}$and $\bar{Q}^{-}$respectively are the actual

integral terms in the PIDE. These two terms are replaced by the approximate sums $\mathbf{J}_{1}$ and $\mathbf{J}_{2}$ respectively as given below:

$$
\begin{array}{r}
\mathrm{J}_{1}=\sum_{i 1=K 1_{i_{1}}^{+}}^{K 2_{i_{1}}^{+}} \sum_{i 2=K 1_{i_{2}}^{+}}^{K 2_{i_{2}}^{+}} \ldots \sum_{i k=K 1_{k}^{+}}^{K 2_{i_{l}}^{+}} u\left(t_{n}, x_{l}+q_{i 1}^{+}, \ldots x_{i k}+q_{i k}^{+}\right)- \\
u\left(t_{n}, x_{i 1}, \ldots x_{i k}\right) \Phi^{+}(\bar{q}+)
\end{array}
$$

The quantities $\Delta t$ and $\Delta x_{i_{l}}$ are the mesh widths defined as :

$\Delta x_{i_{l}}=\frac{\delta_{i_{l}}-\alpha_{i_{l}}}{M}, i_{l}=1,2 \ldots \mathrm{k}$ and $\Delta t=\frac{T}{N}, \mathrm{~T}$ being the time period under consideration. In order to reduce writing the subscripts in the in the finite difference approximation of the derivative terms, the following notation is introduced:

$\mathrm{u}\left(\mathrm{t}_{\mathrm{n}}, \mathrm{x}_{\mathrm{i} 1,} \mathrm{x}_{\mathrm{i} 2, \ldots}, \ldots \mathrm{x}_{\mathrm{il}+1, \ldots} \mathrm{x}_{\mathrm{ik} .}\right)=u_{i_{l}}^{n}$ and $\mathrm{u}\left(\mathrm{t}_{\mathrm{n}+1}, \mathrm{x}_{\mathrm{i} 1,} \mathrm{x}_{\mathrm{i} 2, \ldots}, \ldots \mathrm{x}_{\mathrm{il}, \ldots}, \mathrm{x}_{\mathrm{ik} .}\right)=\underline{u^{n}}$.

Now the system of equations (5) take the form

$$
\begin{aligned}
& c_{i_{1}} \Delta t \underline{u_{i_{1-1}}^{n+1}}+(1-\mathrm{a} \Delta t) \underline{u_{i_{1}}^{n+1}}+b_{i_{1}} \Delta t \underline{u_{i_{1+1}}^{n+1}}+c_{i_{2}} \Delta t \underline{u_{i_{2-1}}^{n+1}} \\
& b_{2} \Delta t \underline{u_{i_{2+1}}^{n+1}}+\ldots \ldots+c_{i_{k}} \Delta t \underline{u_{i_{k+1}}^{n+1}}+b_{i_{k}} \Delta t \underline{u_{i_{k+1}}^{n+1}}=\mathrm{J}_{2}-\mathrm{J}_{1} . \ldots(6),
\end{aligned}
$$

$\mathrm{n}=0,1, \ldots \mathrm{N}-1$ and $u_{i_{l}}^{\mathrm{n}+1}=0$ for $i_{l}>\mathrm{M}$ and with initial condition $\mathrm{u}_{0}^{0}=\mathrm{x}_{0}$

The coefficients in the above equation are defined as

$$
\begin{gathered}
c_{i_{1}}=\frac{H_{i_{l}}}{2\left(\Delta \mathrm{x}_{\mathrm{i}_{1}}\right)^{2}}, \quad b_{i_{l}}=\frac{f_{i_{l}}}{\Delta x_{i_{l}}} \Delta t+c_{i_{l}}, \quad i_{l}=1,2 \ldots \ldots . \mathrm{k} \text { and } \\
\mathrm{a}=\sum_{l=1}^{k}\left(b_{i_{l}}+c_{i_{l}}\right) .
\end{gathered}
$$

With these notations equations (6) can be represented in the matrix form

$$
\widetilde{S} \widetilde{U}^{n}=\mathrm{J}_{2}-\mathrm{J}_{1}
$$

The column vector $\widetilde{U}^{n}$ has its $\mathrm{k}(\mathrm{M}-1)$ components in the order $u_{1, i_{2}, \ldots i_{k}}^{n}, u_{2, i_{2}, \ldots i_{k}}^{n}, \ldots . u_{\mathrm{M}-1, i_{2}, \ldots i_{k}}^{n}, u_{i_{1}, 2, \ldots i_{k}}^{n}, \ldots \ldots \ldots u_{i_{1}, i_{2}, \ldots \mathrm{M}-1}^{n}$.

$\mathrm{S}$ is a block matrix of order $\mathrm{k}(\mathrm{M}-1) \times \mathrm{k}(\mathrm{M}-1)$. It has tri diagonal matrices along the main diagonal and diagonal matrices off the main diagonal. Each block is a square matrix of order (M-1) $\mathrm{x}(\mathrm{M}-1) .0$ denotes the zero matrix of order (M-1) x (M-1).

The diagonal block is $\mathrm{A}$ and each of the blocks in the super diagonal and sub diagonal are the square matrices denoted by $B_{i}$ and $C_{i}$ as noted below:

$$
\begin{aligned}
\mathrm{S}=\left(\begin{array}{cccccc}
A & B_{2} & B_{3} \cdots & B_{M-1} & \mathbf{0} \\
C_{2} & A & B_{2} & \ddots & B_{M-1} \\
C_{3} & C_{2} & A & & \vdots \\
& \vdots & & \vdots & \cdots & B_{2} \\
\mathbf{0} & & C_{M-1} & \cdots & & A
\end{array}\right) \\
\mathrm{A}=\left(\begin{array}{cccccc}
1-\mathrm{a}_{1} \Delta \mathrm{t} & \mathrm{b}_{1} \Delta \mathrm{t} & 0 & & \\
\mathrm{c}_{1} \Delta \mathrm{t} & 1-\mathrm{a}_{1} \Delta \mathrm{t} & \mathrm{b}_{1} \Delta \mathrm{t} & 0 & 0 \\
0 & \mathrm{c}_{1} \Delta \mathrm{t} & 1-\mathrm{a}_{1} \Delta \mathrm{t} & & \vdots \\
& & \vdots & & \ddots & 1-a_{1} \Delta t
\end{array}\right)
\end{aligned}
$$

For $i=2,3, \ldots . M-1$ the matrices $B_{i}$ and $C_{i}$ are the diagonal matrices as shown below:

$$
\mathrm{B}_{\mathrm{i}}=\left(\begin{array}{ccc}
\mathrm{b}_{\mathrm{i}} \Delta \mathrm{t} & 0 \cdots & 0 \\
\vdots & \ddots & \vdots \\
0 & \cdots & \mathrm{b}_{1} \Delta \mathrm{t}
\end{array}\right)
$$




$$
\mathrm{C}_{\mathrm{i}}=\left(\begin{array}{ccc}
\mathrm{c}_{\mathrm{i}} \Delta \mathrm{t} & 0 \cdots & 0 \\
\vdots & \ddots & \vdots \\
0 & \cdots & \mathrm{c}_{1} \Delta \mathrm{t}
\end{array}\right)
$$

The matrix $\mathrm{J}_{2}-\mathrm{J}_{1}$ has the representation,

$$
\left(\begin{array}{c}
v_{0}^{n}+\Delta t\left(v_{0}^{n}+h_{0}{ }^{+n}\right) \\
v_{1}^{n}+\Delta t\left(v_{1}^{n}+h_{1}{ }^{+n}\right) \\
\cdot \\
\cdot \\
\cdot \\
v^{n}+\Delta t\left(v_{N-1}^{n}+h_{N-1}{ }^{+n}\right)
\end{array}\right)
$$

Let $\mathrm{E}$ be all of the difference terms in each equation of the system.

Now we prove the consistency and obtain the condition for the stability of the finite difference scheme.

\section{Convergence, Consistency and Stability of the FD scheme.}

To prove that the finite difference scheme is consistent:

\section{Proposition}

The finite difference scheme defined above is consistent, that is, the error term $\epsilon^{n}$ satisfies $\mid\left(\frac{\partial u}{\partial t}+\right.$ $\boldsymbol{D} u+\boldsymbol{I} u)-\left(\frac{u^{n+1}-\underline{u^{n}}}{\Delta t}+\boldsymbol{E} u+J_{1}-J_{2}\right)|=| \overline{\epsilon^{n}}(\mathrm{tn}, \mathrm{xi} 1, \mathrm{xi} 2 \ldots \ldots \mathrm{xik}) \mid \rightarrow 0$ as $\left(\Delta t, \Delta x_{1}, \Delta x_{2}, \ldots . \Delta x_{k}\right) \rightarrow 0$ at each point $\left(\mathrm{tn}, \mathrm{x}_{\mathrm{i}_{1}}, \mathrm{x}_{\mathrm{i}_{2}} \ldots \ldots \mathrm{x}_{\mathrm{i}_{\mathrm{k}}}\right)$ in the mesh under consideration.

Proof:

By Taylor's theorem, $\underline{u^{n+1}}=\underline{u^{n}}+\Delta \mathrm{t} \frac{\partial u}{\partial t}+\frac{(\Delta t)^{2}}{2 !} \frac{\partial^{2} u}{\partial t^{2}}$ to the second order.

$\left|\frac{\partial u}{\partial t}-\frac{\left(u_{i_{1} i_{2} \ldots i_{k}}^{n+1}-u_{i_{1} i_{2} \ldots i_{k}}^{n}\right)}{\Delta t}\right|=\left|\frac{\partial u}{\partial t}-\frac{u^{n+1}-\underline{u^{n}}}{\Delta t}\right|$

$$
\begin{aligned}
=\mid \frac{\partial u}{\partial t}- & \frac{1}{\Delta t}\left(\Delta \mathrm{t} \frac{\partial u}{\partial t}+\frac{(\Delta t)^{2}}{2 !} \frac{\partial^{2} u}{\partial t^{2}}\right) \mid \\
& =\frac{(\Delta t)^{2}}{2 !}\left\|\frac{\partial^{2} u}{\partial t^{2}}\right\| \\
& \rightarrow 0, \text { as }\left(\Delta t, \Delta x_{1}, \Delta x_{2}, \ldots . \Delta x_{k}\right) \rightarrow 0
\end{aligned}
$$

$\mid \mathrm{Du} \mathrm{u}^{\mathrm{n}}-\mathrm{Eu}(\mathrm{tn}, \mathrm{xi} 1, \mathrm{x}$ i2 $\ldots \ldots \mathrm{xik}) \mid=$ $\mid \mathrm{Du}^{\mathrm{n}+1}+\Delta \mathrm{tD}(\hat{\mathrm{t}}, \mathrm{xi} 1, \mathrm{x}$ i2 $\ldots \ldots \mathrm{xik})-\mathrm{Eu}(\mathrm{tn}, \mathrm{xi} 1, \mathrm{x} \mathrm{i} 2 \ldots \ldots \mathrm{xik}) \mid$, where $\hat{\mathrm{t}}$ lies in the interval $\left[\mathrm{t}_{\mathrm{n}}, \mathrm{t}_{\mathrm{n}+1}\right]$.

For any one of the components $\mathrm{x}_{\mathrm{il}}$,

$\left|f_{i l} \frac{\partial u}{\partial x_{i}}-\mathrm{f}_{\mathrm{il}} \frac{\left(u_{i_{1} i_{2} \ldots \mathrm{i}_{1+1, \ldots}}^{n+1} i_{k}-u_{i_{1} i_{2} \ldots \mathrm{i}_{1, . .} i_{k}}^{n+1}\right)}{\Delta t}\right|=\left|\mathrm{f}_{\mathrm{il}} \frac{\partial u}{\partial x_{i}}-\mathrm{f}_{\mathrm{il}} \frac{u_{i_{l+1}}{ }^{n+1}-u_{i_{l}}^{n+1}}{\Delta x_{i}}\right|$

$\frac{u_{i_{l+1}}^{n+1}-u_{i_{l}}^{n+1}}{\Delta x_{i}}=\frac{\partial u}{\partial x_{i}}+\frac{\Delta x_{i_{l}}}{2 !} \frac{\partial^{2} u}{\partial x_{i_{l}}^{2}}+\ldots$

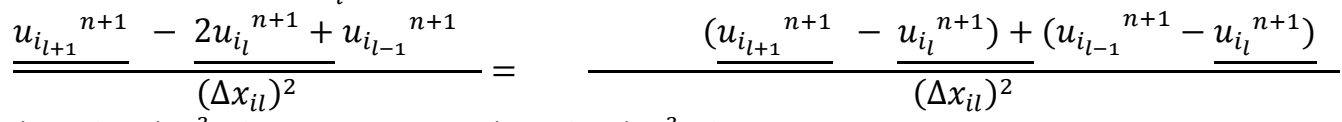

$=\left(\frac{\partial u}{\partial x_{i l}}+\frac{\Delta x_{i_{l}}}{2 !} \frac{\partial^{2} u}{\partial x_{i_{l}}{ }^{2}}+\frac{\Delta x_{i_{l}}{ }^{2}}{3 !} \frac{\partial^{3} u}{\partial x_{i_{l}}{ }^{3}}+..\right)+\left(-\frac{\partial u}{\partial x_{i l}}+\frac{\Delta x_{i_{l}}}{2 !} \frac{\partial^{2} u}{\partial x_{i_{l}}{ }^{2}} \frac{\Delta x_{i_{l}}{ }^{2}}{3 !} \frac{\partial^{3} u}{\partial x_{i_{l}}{ }^{3}}+..\right)$

$=\Delta x_{i_{l}} \frac{\partial^{2} u}{\partial x_{i_{l}}{ }^{2}}+2 \frac{\Delta x_{i_{l}}{ }^{3}}{4 !} \frac{\partial^{4} u}{\partial x_{i_{l}}{ }^{4}}+\ldots$

So $\left|\mathrm{Du}^{\mathrm{n}+1}+\Delta \mathrm{tD}\left(\hat{\mathrm{t}}, \mathrm{x}_{\mathrm{i}_{1}}, \mathrm{x}_{\mathrm{i}_{2}} \ldots \ldots \mathrm{x}_{\mathrm{i}_{\mathrm{k}}}\right)-\operatorname{Eu}\left(\mathrm{tn}, \mathrm{x}_{\mathrm{i}_{1}}, \mathrm{x}_{\mathrm{i}_{2}} \ldots \ldots \mathrm{x}_{\mathrm{i}_{\mathrm{k}}}\right)\right|$

$\leq \Delta \mathrm{t}\left\|\frac{\partial u}{\partial t}\right\|+\sum_{l=1}^{k}\left[f_{i_{l}}\left(\frac{\Delta x_{i_{l}}}{2 !} \frac{\partial^{2} u}{\partial x_{i_{l}}{ }^{2}}\right)+\frac{H_{i_{l}}}{2}\left(\Delta x_{i_{l}} \frac{\partial^{2} u}{\partial x_{i_{l}}{ }^{2}}+2 \frac{\Delta x_{i_{l}}{ }^{3}}{4 !} \frac{\partial^{4} u}{\partial x_{i_{l}}{ }^{4}}\right)\right.$

$\leq \Delta \mathrm{t}\left\|\frac{\partial u}{\partial t}\right\|+\sum_{l=1}^{k}\left[\left|f_{i_{l}}\right| \frac{\Delta x_{i_{l}}}{2 !}|| \frac{\partial^{2} u}{\partial x_{i_{l}}{ }^{2}}\left\|+\left|\frac{H_{i_{l}}}{2}\right| \Delta x_{i_{l}}|| \frac{\partial^{2} u}{\partial x_{i_{l}}{ }^{2}}\right\|\right.$

$\rightarrow 0$ as $\left(\Delta \mathrm{t}, \Delta x_{1}, \Delta x_{i_{2}}, \ldots \Delta x_{i_{k}}\right) \rightarrow 0$....(ii)

For the integral term $\mathrm{I}_{1}$ and its approximation $\mathrm{J}_{1}$, $\left|\mathrm{I}_{1}-\mathrm{J}_{1}\right|$ 


$$
\begin{gathered}
=\mid \hat{\lambda}\left(\int_{\hat{Q_{+}^{+}}}\left[\bar{u}\left(x+h\left(\overline{x_{o}}, t, \bar{q}\right)\right)-\bar{u}\left(\overline{x_{o}}, t\right)\right] \Phi^{+}(\bar{q}+) d \overline{q^{+}}\right)- \\
\quad\left(\sum_{i 1=K 1_{i_{1}}^{+}}^{K 2_{i_{1}}^{+}} \sum_{i 2=K 1_{i_{2}}^{+}}^{K 2_{i_{2}}^{+}} \ldots \sum_{i k=K 1_{k}^{+}}^{K 2_{i_{l}}^{+}} u\left(t_{n}, x_{i 1}+q_{i 1}^{+}, \ldots x_{i k}+q_{i k}^{+}\right)-\right. \\
\left.u\left(t_{n}, x_{i 1}, \ldots x_{i k}\right) \Phi^{+}(\bar{q}+)\right) \mid
\end{gathered}
$$

For the sake of simplicity of notations considering $\mathrm{k}=2$ and taking $\bar{x}=(\mathrm{x}, \mathrm{y})$,

$\bar{q}=\left(\mathrm{q}_{\mathrm{x}}, \mathrm{q}_{\mathrm{y}}\right), \quad\left|\mathrm{I}_{1}-\mathrm{J}_{1}\right|$

$$
\begin{aligned}
& \left.=|\hat{\lambda}| \mid \sum_{i=K 1_{x}^{+}}^{K 2_{x}^{+}} \sum_{j=K 1_{y}^{+}}^{K 2_{y}^{+}}\left[\cdot u_{a+i, b+j}^{n}-u_{i, j}^{n}\right] \Phi^{+}\left(q_{i}^{+}, q_{j}^{+}\right)\right)- \\
& \quad \iint_{\hat{Q}}\left[\cdot u\left(t_{n}, x_{i}+q_{x}^{+}, y_{j}+q_{y}^{+}\right)-u\left(t_{n}, x_{i,}, y_{j}\right)\right] \Phi^{+}(\bar{q}+) d \overline{q^{+}} \mid \\
& \left.=|\hat{\lambda}| \mid \sum_{i=K 1_{x}^{+}}^{K 2_{x}^{+}} \sum_{j=K 1_{y}^{+}}^{K 2_{1}^{+}} \cdot u_{a+i, b+j}^{n} \Phi^{+}\left(q_{i}^{+}, q_{j}^{+}\right)\right)- \\
& \qquad \iint_{\hat{Q}}\left[\cdot u\left(t_{n}, x_{i}+q_{x}^{+}, y_{j}+q_{y}^{+}\right) \Phi^{+}(\bar{q}+) d \overline{q^{+}},\right.
\end{aligned}
$$

Since $\left.\sum_{i=K 1_{x}^{+}}^{K 2_{x}^{+}} \sum_{j=K 1_{y}^{+}}^{K 2_{y}^{+}} u_{i, j}^{n} \Phi^{+}\left(q_{x}^{+}, q_{y}^{+}\right)\right) \approx \iint_{\hat{Q}}\left[\cdot u\left(t_{n}, x_{i}, y_{j}\right) \Phi^{+}(\bar{q}+) d \overline{q^{+}}\right.$

$$
\begin{aligned}
=|\hat{\lambda}| \mid \sum_{i=K 1_{x}^{+}}^{K 2_{x}^{+}} \sum_{j=K 1_{y}^{+}}^{K 2_{y}^{+}} \int_{\left(i-\frac{1}{2}\right) \Delta x}^{\left(i+\frac{1}{2}\right) \Delta x} \int_{\left(j-\frac{1}{2}\right) \Delta y}^{\left(j+\frac{1}{2}\right) \Delta y}\left[u_{a+i, b+j}^{n} \Phi^{+}\left(q_{i}^{+}, q_{j}^{+}\right)-\right. \\
u\left(t_{n}, x_{i}+q_{x}^{+}, y_{j}+q_{y}^{+}\right) \Phi^{+}\left(q_{x}^{+}, q_{y}^{+}\right) d q_{x} q_{y} \mid
\end{aligned}
$$

$\leq \widehat{\lambda}\left|\sum_{i=K 1_{x}^{+}}^{K 2_{x}^{+}} \sum_{j=K 1_{y}^{+}}^{K 2_{y}^{+}} \int_{\left(i-\frac{1}{2}\right) \Delta x}^{\left(i+\frac{1}{2}\right) \Delta x} \int_{\left(j-\frac{1}{2}\right) \Delta y}^{\left(j+\frac{1}{2}\right) \Delta y}\right|\left[\left(q_{i}^{+}-q_{x}^{+}\right) \frac{\partial u}{\partial x}+\left(q_{j}^{+}-q_{y}^{+}\right) \frac{\partial u}{\partial y}\right] \Phi^{+} \mathrm{d} q_{x} q_{y}$

$\leq \widehat{\lambda} \mid\left[\Delta \mathrm{x}\left\|\frac{\partial u}{\partial x}\right\|+\Delta \mathrm{y}\left\|\frac{\partial u}{\partial y}\right\|\right] \sum_{i=K 1_{x}^{+}}^{K 2_{x}^{+}} \sum_{j=K 1_{y}^{+}}^{K 2_{y}^{+}} \int_{\left(i-\frac{1}{2}\right) \Delta x}^{\left(i+\frac{1}{2}\right) \Delta x} \int_{\left(j-\frac{1}{2}\right) \Delta y}^{\left(j+\frac{1}{2}\right) \Delta y} d q_{x} q_{y}$

$\leq \widehat{|\lambda|}\left[\Delta \mathrm{x}\left\|\frac{\partial u}{\partial x}\right\|+\Delta \mathrm{y}\left\|\frac{\partial u}{\partial y}\right\|\right]\left(K 2_{x}^{+}-K 1_{x}^{+}\right)\left(K 2_{y^{-}}^{+} K 1_{y}^{+}\right)$

$\rightarrow 0$ as $\left(\Delta \mathrm{t}, \Delta x_{1}, \Delta x_{i_{2}}, \ldots \Delta x_{i_{k}}\right) \rightarrow 0$.

Similarly it can be proved $\left|\mathrm{I}_{2}-\mathrm{J}_{2}\right| \rightarrow 0$ as $\left(\Delta \mathrm{t}, \Delta x_{1}, \Delta x_{i_{2}}, \ldots \Delta x_{i_{k}}\right) \rightarrow 0$

From (i), (ii) and (iii) the required result follows.

\section{To find the condition for the stability of the scheme:}

For any square matrix $\mathrm{A}$ of order $\mathrm{n}$, the norm of the matrix is defined as the maximum of the sum of the absolute values of any row (column) and is written as $\|\mathrm{A}\|$. Also the spectral radius of $\mathrm{A}$ is defined as $\rho$ (A) $=\max _{1 \leq i \leq n}\left|\lambda_{i}\right|$, where $\lambda_{i}(\mathrm{i}=1,2 . . \mathrm{n})$ are the eigenvalues of the matrix A. Moreover it is known that $\rho(\mathrm{A}) \leq \| \mathrm{A}$ $\|$ and the condition $\rho(\mathrm{A}) \leq 1$ ensures the stability of the scheme.

So the finite difference scheme is stable if the spectral radius is not greater than 1 .

For the block matrix $\mathrm{S}$ in equation (7) its norm is given by,

$$
\begin{array}{r}
\|\mathrm{A}\|=|1-\mathrm{a} \Delta \mathrm{t}|+\sum_{i=2}^{M-1}\left(\left|b_{i}\right|+\left|c_{i}\right|\right) \Delta t \text { and }\|\mathrm{A}\| \leq 1 \text { gives the condition, } \\
|1-\mathrm{a} \Delta \mathrm{t}|+\sum_{i=2}^{M-1}\left(\left|b_{i}\right|+\left|c_{i}\right|\right) \Delta t \leq 1 \quad \ldots .(8) \\
\text { But }|\mathrm{a} \Delta \mathrm{t}|-1 \leq|1-\mathrm{a} \Delta \mathrm{t}|
\end{array}
$$

So inequality (8) can be written as $|\mathrm{a} \Delta \mathrm{t}|-1 \leq|1-\mathrm{a} \Delta \mathrm{t}|$

Hence $\Delta \mathrm{t} \leq \frac{2}{|a|}$.

Thus the condition for stability of the scheme is $\Delta t \leq \frac{2}{|a|}$.

\section{Convergence}

According to Lax Ritchmeyer theorem the numerical solution of any well-posed problem converges to the actual solution provided that the scheme is consistent and stable[2],[4]. Thus the convergence of the finite difference scheme that has been defined for the system of equations (8) is convergent.

\section{To verify diagonal dominance for the matrix S:}

The system of equations (8) will have a solution and the solution will be unique if the entries of the matrix exhibit diagonal dominance[2].

For diagonal dominance it should be true that

$$
\sum_{i=1}^{M-1}\left(\left|b_{i}\right|+\left|c_{i}\right|\right) \Delta t \leq|1-\mathrm{a} \Delta \mathrm{t}|
$$




$$
\begin{aligned}
& \leq 1+|\mathrm{a} \Delta \mathrm{t}| \\
& \leq 1+\sum_{i=1}^{M-1}\left(\left|b_{i}\right|+\left|c_{i}\right|\right) \Delta t
\end{aligned}
$$

i.e $\sum_{i=1}^{M-1}\left(\left|b_{i}\right|+\left|c_{i}\right|\right) \Delta t \leq 1+\sum_{i=1}^{M-1}\left(\left|b_{i}\right|+\left|c_{i}\right|\right) \Delta t$, which is always true.

Hence the solution is unique.

\section{Conclusion}

We know that stochastic models play important roles in elucidating many areas of engineering sciences, environmental sciences and quantitative finance. They can also be used to analyze the variability inherent in biological and medical processes, or deal with uncertainties affecting managerial decisions. The underlying stochastic process may depend on several factors. We can identify these factors and also choose a suitable statistical distribution to represent the jump terms. Then our multi dimensional model can be applied and tested for an appropriate problem.

\section{References}

[1]. R.Cont \& Ekaterina Voltchkova,"A finite difference scheme for option pricing in jump diffusion and exponential Levy models", Rapport Interne CMAP No., 513,(Sep2003).

[2]. David G.Feingold \& R.S.Varga, "Block diagonally dominant matrices and generalizations of the Gerschgorin circle theorem", Pacific J.of mathematics, vol 12 no. 4 (1962).

[3]. Oskendal.B, "Stochastic differential equations- an introduction with applications", 5 th ed, Springer Verlag (2000).

[4]. A.K.Pani et al, "Numerical methods for hyperbolic and parabolic integro-differential equations", J.of integral equations and applications, vol 4 (4) 533-584, (summer 1992).

[5]. F.B.Hanson, “Applied stochastic processes and control for jump diffusions-modelling, analysis and computations", SIAM (2007). 